\title{
NODE FAILURE TIME ANALYSIS FOR MAXImum STABILITY VS MiNimum DistanCE SPANNING TREE BASED DATA GATHERING IN MOBILE SENSOR NETWORKS
}

\author{
Natarajan Meghanathan ${ }^{1}$ and Philip Mumford ${ }^{2}$ \\ ${ }^{1}$ Jackson State University, 1400 Lynch St, Jackson, MS, USA \\ ${ }^{2}$ Air Force Research Lab/RYWC, Wright-Patterson Air Force Base, OH, USA \\ Corresponding Author E-mail: natarajan.meghanathan@jsums . edu
}

\begin{abstract}
A mobile sensor network is a wireless network of sensor nodes that move arbitrarily. In this paper, we explore the use of a maximum stability spanning tree-based data gathering (Max.Stability-DG) algorithm and a minimum-distance spanning tree-based data gathering (MST-DG) algorithm for mobile sensor networks. We analyze the impact of these two algorithms on the node failure times, specifically with respect to the node lifetime (the time of first node failure) and network lifetime (the time of disconnection of the network of live sensor nodes due to one or more node failures). Both the Max.Stability-DG and MST-DG algorithms are based on a greedy strategy of determining a data gathering tree when one is needed and using that tree as long as it exists. The Max.Stability-DG algorithm assumes the availability of the complete knowledge of future topology changes and determines a data gathering tree whose corresponding spanning tree would exist for the longest time since the current time instant; whereas, the MST-DG algorithm determines a data gathering tree whose corresponding spanning tree is the minimum distance tree at the current time instant. We observe a node lifetime - network lifetime tradeoff: the Max.Stability-DG trees incur a lower node lifetime due to repeated use of a data gathering tree for a longer time; on the other hand, the Max.Stability$D G$ trees incur a longer network lifetime.
\end{abstract}

\begin{abstract}
KEYWORDS
Data Gathering, Maximum Stability, Minimum Distance Spanning Tree, Mobile Sensor Networks, Node Lifetime, Coverage Loss Time
\end{abstract}

\section{INTRODUCTION}

A mobile sensor network is a dynamically changing wireless distributed system of arbitrarily moving sensor nodes that operate under limited battery charge, memory and processing capacity. The common objective of many of the data gathering algorithms for the static sensor networks has been to conserve energy and maximize the node lifetime and network lifetime. In this context, in a recent research [5], we evaluated the performance of the data gathering algorithms based on different communication topologies and observed the minimum distance-spanning tree based data gathering (MST-DG) trees to be the most energy-efficient. However, with mobility, the network 
topology changes dynamically with time and thus, there is a need to determine stable data gathering trees that do not break frequently.

In the first half of the paper, we describe our benchmarking algorithm for maximum stability data gathering (Max.Stability-DG) in mobile sensor networks such that the number of tree discoveries is the global minimum. Given the complete knowledge of the future topology changes, the Max.Stability-DG algorithm operates based on the following greedy principle: Whenever a data gathering tree is required at time instant $t$, choose the longest-living data gathering tree from $t$. The above strategy is repeated over the duration of the data gathering session. The sequence of such longest-living data gathering trees incurs the minimum number of tree discoveries. The worst-case run-time complexity of the Max.Stability-DG tree algorithm is $\mathrm{O}\left(n^{2} T \log n\right)$ and $\mathrm{O}\left(n^{3} T \log n\right)$ when operated under sufficient-energy and energy-constrained scenarios respectively, where $n$ is the number of nodes in the network and $T$ is the total number of rounds of data gathering; $\mathrm{O}\left(n^{2} \log n\right)$ is the worst-case run-time complexity of the minimum-weight spanning tree algorithm (we use Prim's algorithm [6]) used to determine the underlying spanning trees from which the data gathering trees are derived. A similar approach is adopted to determine the sequence of MST-DG trees - with the only difference being that the underlying spanning tree is a minimum distance spanning tree determined based on the local network topology and not at the future topology changes.

In the second half of the paper, we conduct an exhaustive simulation study of the Max.StabilityDG trees vs. the MST-DG trees and analyze their impact on the node lifetime, network lifetime and coverage loss time. To the best of our knowledge, we could not find any such comprehensive analysis of two data gathering strategies for mobile sensor networks and also with respect to the node failure times beyond the first node failure. The rest of the paper is organized as follows: Section 2 presents the algorithms to determine the Max.Stability-DG trees and MST-DG trees. Section 3 presents the simulation environment used and introduces the performance metrics. Section 4 describes the simulation results observed for the node and network lifetime. Section 5 concludes the paper.

\section{Data Gathering Algorithms Based On Maximum Stability and Minimum Distance SPANNING TREeS}

The Max.Stability-DG algorithm is based on a greedy look-ahead principle and the intersection strategy of static graphs. When a mobile data gathering tree is required at a sampling time instant $t_{i}$, the strategy is to find a mobile graph $G(i, j)=G_{i} \cap G_{i+1} \cap \ldots G_{j}$ such that there exists a spanning tree in $G(i, j)$ and no spanning tree exists in $G(i, j+1)=G_{i} \cap G_{i+1} \cap \ldots G_{j} \cap G_{j+1}$. We find such an epoch $t_{i}, \ldots, t_{j}$ as follows: Once a mobile graph $G(i, j)$ is constructed with the edges assigned the weights corresponding to the geometric mean of the weights in the constituent static graphs $G_{i}, G_{i+1}, \ldots, G_{j}$, we run the Prim's minimum-weight spanning tree algorithm on the mobile graph $G(i, j)$. If $G(i, j)$ is connected, we will be able to find a spanning tree in it. We repeat the above procedure until we reach a mobile graph $G(i, j+1)$ in which no spanning tree exists and there existed a spanning tree in $G(i, j)$. It implies that a spanning tree basically existed in each of the static graphs $G_{i}, G_{i+1}, \ldots, G_{j}$ and we refer to it as the mobile spanning tree for the time instants $t_{i}, \ldots, t_{j}$. To obtain the corresponding mobile data gathering tree, we choose an arbitrary root node for this mobile spanning tree and run the Breadth First Search (BFS) algorithm on it starting from the root node. The direction of the edges in the spanning tree and the parent-child relationships are set as we traverse its vertices using BFS. The resulting mobile data gathering tree with the chosen root node (as the leader node) is used for every round of data gathering spanning time instants $t_{i}, \ldots, t_{j}$. We then set $i=j+1$ and repeat the above procedure to find a mobile spanning tree and its corresponding mobile data gathering tree that exists for the maximum amount of time since $t_{j+1}$. We repeat this procedure to obtain a sequence of such maximum lifetime (i.e., longestliving) mobile data gathering trees over the timescale of the data gathering session. While 
operating the algorithm under energy-constrained scenarios, one or more sensor nodes may die due to exhaustion of battery charge even though the underlying spanning tree may topologically exist. For example, if we have determined a data gathering tree spanning across time instants $t_{i}$ to $t_{j}$ using the above approach, and we come across a time instant $t_{k}(i \leq k \leq j)$ at which a node in the tree fails, we simply restart the Max.Stability-DG algorithm starting from time instant $t_{k}$ considering only the live sensor nodes (i.e., the sensor nodes that have positive available energy) and determine the longest-living data gathering tree that spans all the live sensor nodes since $t_{k}$.

We compare the performance of the Max.Stability-DG trees with that of the minimum-distance spanning tree based data gathering (MST-DG) trees. The sequence of MST-DG trees for the duration of the data gathering session is generated as follows: If a MST-DG tree is not known for a particular round, we run the Prim's minimum-weight spanning tree algorithm on the static graph representing the snapshot of the network topology generated at the time instant corresponding to the round. Since the weights of the edges in a static graph represent the physical Euclidean distance between the constituent end nodes of the edges, the Prim's algorithm will return the minimum-distance spanning tree on the static graph. We then choose an arbitrary root node and run the Breadth First Search (BFS) algorithm starting from this node. The MST-DG tree is the rooted form of the minimum-distance spanning tree with the chosen root node as the leader node. We continue to use the MST-DG tree as long as it exists. The leader node of the MST-DG tree remains the same until the tree breaks due to node mobility or node failures. When the MST-DG tree ceases to exist for a round, we repeat the above procedure. In a recent work [5], we have observed the minimum-distance spanning tree-based data gathering trees to be the most energyefficient communication topology for data gathering in static sensor networks.

\section{Simulation Environment And Performance Metrics}

The simulations are conducted in a discrete-event simulator developed (in Java) by us exclusively for data gathering in mobile sensor networks. The MAC (medium access control) layer is assumed to be collision-free and considered an ideal channel without any interference. Sensor nodes are assumed to be both TDMA (Time Division Multiple Access) and CDMA (Code Division Multiple Access)-enabled [11]. The network dimension is $100 \mathrm{~m} \times 100 \mathrm{~m}$. The number of nodes in the network is 100 and initially, the nodes are uniform-randomly distributed throughout the network. The sink is located at $(50,300)$, outside the network field. For a given simulation run, the transmission range per sensor node is fixed and is the same across all nodes. The network density is varied by varying the transmission range per sensor node of $25 \mathrm{~m}$ (representative of moderate density, with connectivity of $97 \%$ and above) and 40m (high density, with $100 \%$ connectivity).

Each node is supplied with limited initial energy ( $2 \mathrm{~J}$ per node) and the simulations are conducted until the network of live sensor nodes gets disconnected due to the failures of one or more nodes. The energy consumption model used is a first order radio model [12] that has been also used in several of the well-known previous work (e.g., [1][2]) in the literature. According to this model, the energy expended by a radio to run the transmitter or receiver circuitry is $E_{\text {elec }}=50 \mathrm{~nJ} / \mathrm{bit}$ and $\epsilon_{a m p}=100 \mathrm{pJ} / \mathrm{bit} / \mathrm{m}^{2}$ for the transmitter amplifier. The radios are turned off when a node wants to avoid receiving unintended transmissions. The energy lost in transmitting a $k$-bit message over a distance $d$ is given by: $E_{T X}(k, d)=E_{\text {elec }}{ }^{*} k+\epsilon_{\text {amp }}{ }^{*} k^{*} d^{2}$. The energy lost to receive a $k$-bit message is: $E_{R X}(k)=E_{\text {elec }} * k$.

We conduct constant-bit rate data gathering at the rate of 4 rounds per second (one round for every 0.25 seconds). The size of the data packet is 2000 bits; the size of the control messages used for tree discoveries is assumed to be 400 bits. We assume that a tree discovery requires 
network-wide flooding of the 400-bit control messages such that each sensor node will broadcast the message exactly once in its neighborhood. As a result, each sensor node will lose energy to transmit the 400-bit message over its entire transmission range and receive the message from each of its neighbor nodes. The node mobility model used is the well-known Random Waypoint mobility model [13] with the maximum node velocity $\left(v_{\max }\right)$ being $3 \mathrm{~m} / \mathrm{s}, 10 \mathrm{~m} / \mathrm{s}$ and $20 \mathrm{~m} / \mathrm{s}$ representing scenarios of low, moderate and high mobility respectively.

We generated 200 mobility profiles of the network for a total duration of 6000 seconds, for every combination of $v_{\max }$ and the number of static nodes. Every data point in the results presented in Figures 1 through 4 is averaged over these 200 mobility profiles. The performance metrics measured in the simulations are: (i) Node Lifetime - measured as the time of first node failure due to exhaustion of battery charge. (ii) Network Lifetime - measured as the time of disconnection of the network of live sensor nodes, while the network would have stayed connected if all the nodes were alive at that time instant.

We obtain the distribution of node failures as follows: The probability for ' $x$ ' number of node failures ( $x$ from ranging from 1 to 100 as we have a total of 100 nodes in our network for all the simulations) for a given combination of the operating conditions is measured as the number of mobility profile files that reported $x$ number of node failures divided by 200 , which is the total number of mobility profiles used for every combination of maximum node velocity and number of static nodes. Similarly, we keep track of the time at which ' $x$ ' ( $x$ ranging from 1 to 100) number of node failures occurred in each of the 200 mobility profiles for a given combination of operating conditions and the values for the time of node failures reported in Figures 3 and 4 are an average of these data collected over all the mobility profile files.

\section{NODE LIFETIME AND NETWORK LIFETIME}

We observe a tradeoff between node lifetime and network lifetime for maximum stability vs. minimum-distance spanning tree based data gathering in mobile sensor networks. The MST-DG trees incur larger node lifetimes for all the 48 operating combinations of maximum node velocity, number of static nodes and transmission range per node. The Max.Stability-DG trees incur larger network lifetime for most of the operating conditions. The lower node lifetime incurred with the Max.Stability-DG trees is attributed to the unfairness in node usage resulting due to the continued use of stable data gathering trees for a longer time and to the approach of not changing the leader node and the intermediate aggregator nodes as long as a tree exists (such an approach is adopted to reduce the overall message complexity and network-wide energy consumption). Max.StabilityDG trees incur a significant increase in node lifetime, with increase in the maximum node velocity, especially when operated in moderate transmission ranges per node. The percentage increase in node lifetime for the MST-DG trees with increase in node mobility is relatively much lower.
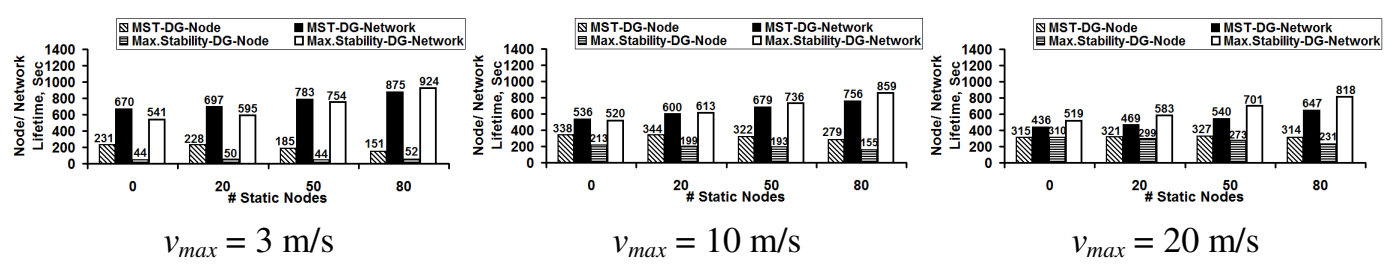

Figure 1: Average Node and Network Lifetime (Transmission Range $=25 \mathrm{~m}$ )

The node lifetime incurred for the MST-DG trees can be larger than that of the Max.Stability-DG trees by as large as $400 \%$ at low and moderate levels of node mobility and by as large as $135 \%$ at higher levels of node mobility. For a given level of node mobility, the difference in the node lifetimes incurred for the MST-DG trees and Max.Stability-DG trees increases with increase in 
the transmission range per node (for a fixed number of static nodes) and either remains the same or slightly increases with increase in the number of static nodes (for a fixed transmission range per node). At larger transmission ranges per node, the data gathering trees are bound to be more stable, and the negative impact of this on node lifetime is significantly felt in the case of the Max.Stability-DG trees.
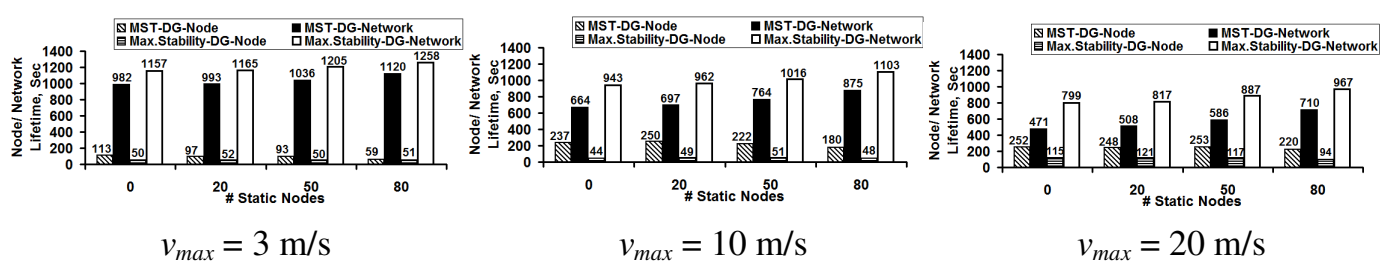

Figure 2: Average Node and Network Lifetime (Transmission Range $=40 \mathrm{~m}$ )

The difference in the network lifetime incurred for the Max.Stability-DG trees and that of the MST-DG trees increases with increase in the maximum node velocity and transmission range per node. We observe the network lifetime incurred with the two data gathering trees increases with increase in the number of static nodes for a given value of $v_{\max }$ and transmission range per node. For a given level of node mobility, the network lifetime increases with increase in transmission range per node; however, for the MST-DG trees, the rate of increase decreases with increase in the maximum node velocity. This could be attributed to the relative instability of the MST-DG trees at high node mobility levels, requiring frequent tree reconfigurations. During a networkwide flooding, all nodes in the network tend to lose energy, almost equally. The Max.StabilityDG trees maintain a steady increase in the network lifetime with increase in transmission range per node for all levels of node mobility. For a given transmission range per node and number of static nodes, the network lifetime incurred for the two data gathering trees decreases with increase in the maximum node velocity, especially for the MST-DG trees due to their instability.

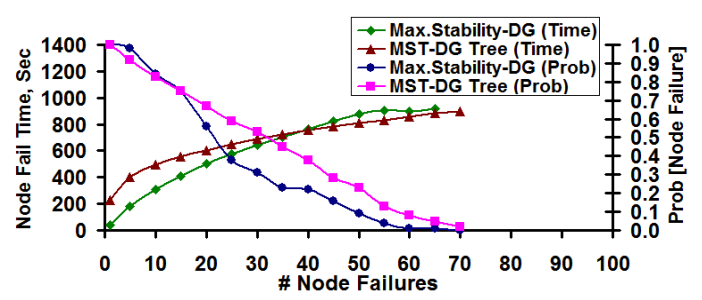

Trans. Range: 25 m, 0 static nodes

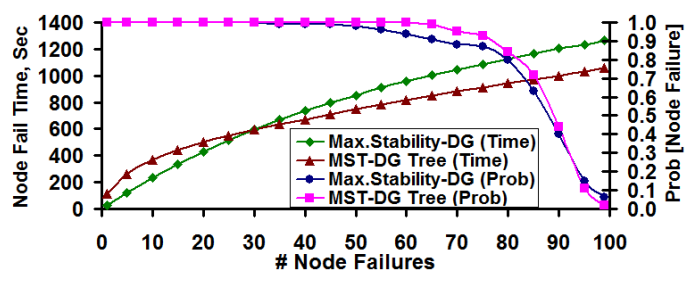

Trans. Range: 40 m, 0 static nodes

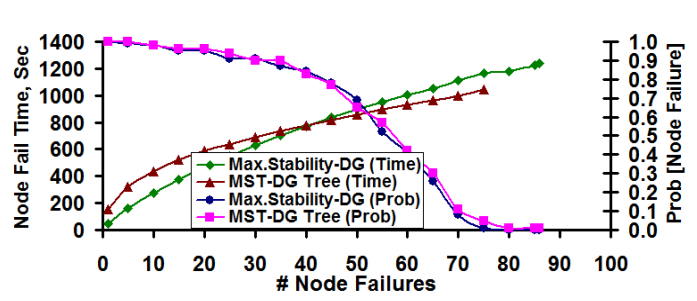

Trans. Range: 25 m, 80 static nodes

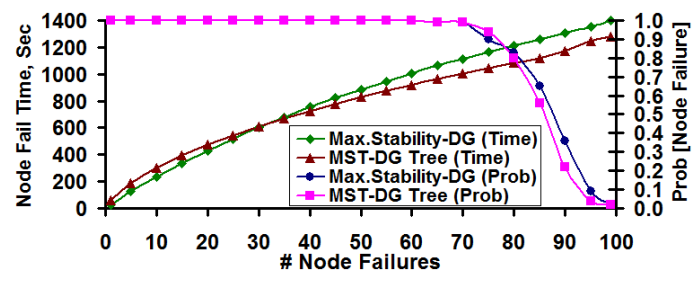

Trans. Range: 40 m, 80 static nodes

Figure 3: Node Failure Times and Probability of Node Failures $\left[v_{\max }=3 \mathrm{~m} / \mathrm{s}\right.$ ]

For a given transmission range per node, the maximum increase in the absolute time of node failures occurs at low node mobility, due to fewer number of energy consuming network-wide flooding based tree discoveries. Since all nodes are likely to lose the same amount of energy with 
flooding, the more we conduct flooding, the larger is the network-wide energy consumption. Even though operating the network at moderate and high levels of node mobility helps us to extend the time of first node failure, the subsequent node failures occur too soon after the first node failure. This could be justified with the observation of flat curves for the MST-DG trees with respect to the distribution of node failure times (in Figures 3 and 4).

The Max.Stability-DG trees compensate for the premature failures of certain nodes by incurring a lower energy loss per round and energy loss per node due to lower tree discoveries and shorter tree height with more even distribution of the number of child nodes per intermediate node. The distribution of node failure times is relatively steeper for the Max.Stability-DG trees. Aided with node mobility, it is possible for certain energy-rich nodes (that might have been leaf nodes in an earlier data gathering tree) to keep the network connected for a longer time by serving as intermediate nodes, and the energy-deficient nodes serve as leaf nodes during the later rounds.

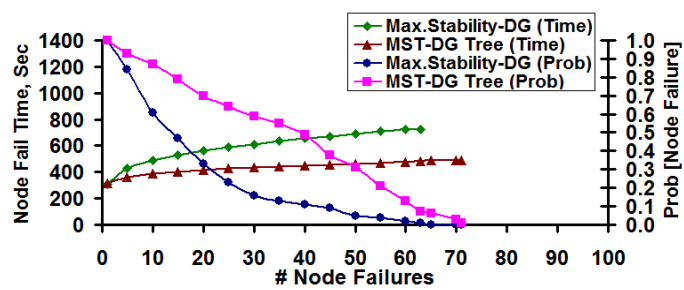

Trans. Range: 25 m, 0 static nodes

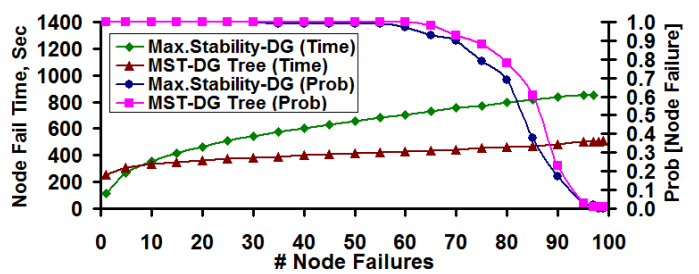

Trans. Range: 40 m, 0 static nodes

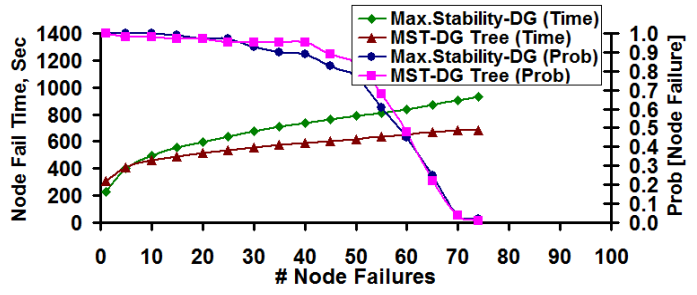

Trans. Range: 25 m, 80 static nodes

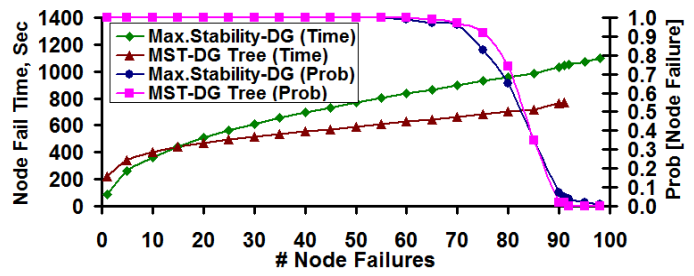

Trans. Range: 40 m, 80 static nodes

Figure 4: Node Failure Times and Probability of Node Failures $\left[v_{\max }=20 \mathrm{~m} / \mathrm{s}\right.$ ]

We observe a lower probability of node failure observed for the Max.Stability-DG trees in comparison to the MST-DG trees when there are 0 static nodes. With the use of static nodes, even though the absolute magnitude of the network lifetime can be marginally increased (by about 10$70 \%$; the increase is larger at moderate transmission range per node and larger values of $v_{\max }$ ), the probability of node failures to occur also increases. For a given transmission range per node and maximum node velocity, we observe the difference between the node lifetime and network lifetime for the Max.Stability-DG trees to increase significantly with increase in the number of static nodes. For a given level of node mobility, we observe the difference in the node lifetime and network lifetime for the Max.Stability-DG trees to increase with increase in the transmission range per node. Relatively, the MST-DG trees incur a very minimal increase in the network lifetime compared to the node lifetime, especially when operated at higher levels of node mobility.

One can also observe from Figures 3 and 4 that the number of node failures that require for the node failure time incurred with the Max.Stability-DG trees to exceed that of the node failure time incurred with the MST-DG trees decreases with increase in maximum node mobility. This could be attributed to the premature very early node failure occurring for the Max.Stability-DG trees when operated under low node mobility scenarios, with the time of first node failure for the MST- 
DG tree being as large as $400 \%$ more than the time of first node failure for the Max.Stability-DG tree. On the other hand, at high levels of node mobility, the time of first node failure incurred with the MST-DG trees is at most 100\% larger than that of the Max.Stability-DG trees. At the same time, the probability for node failures to occur with the Max.Stability-DG trees converges to that of the MST-DG trees when operated at higher levels of node mobility as well as with larger transmission ranges per node. For a given $v_{\max }$ and transmission range per node, the number of node failures required for the failure times incurred with the Max.Stability-DG trees exceeds that of the MST-DG trees with increase in the number of static nodes.

\section{CONCLUSIONS}

We observe a node lifetime - network lifetime tradeoff for data gathering in mobile sensor networks. The minimum-distance spanning trees incur a significantly longer node lifetime that is as large as $400 \%$ compared to the maximum stability-based data gathering (Max.Stability-DG) trees. On the other hand, the Max.Stability-DG trees incur a longer network lifetime that can be as large as $60 \%$ more compared to the MST-DG trees. The MST-DG trees suffer from an avalanche of node failures after the first node failure due to the larger but equal, energy consumption of nodes across the network. As a result, even though the time of first node failure is prolonged, the subsequent node failures happen quickly. The Max.Stability-DG trees, with the tendency to use a tree as long as it exists, burn out the energy supplies at selected nodes; but, the variations in node usage help to increase the time between successive node failures. As part of future work, we will investigate whether energy-aware stability-based data gathering can prolong the time of first node failure.

\section{ACKNOWLEDGMENTS}

This research was sponsored by the U. S. Air Force Office of Scientific Research (AFOSR) through the Summer Faculty Fellowship Program, conducted in June-July 2012 at the U. S. Air Force Research Lab (AFRL), Wright-Patterson Air Force Base (WPAFB) Dayton, OH. The PA approval number is $88 \mathrm{ABW}-2012-4893$.

\section{REFERENCES}

[1] S. Lindsey, C. Raghavendra and K. M. Sivalingam, "Data Gathering Algorithms in Sensor Networks using Energy Metrics," IEEE Transactions on Parallel and Distributed Systems, 13(9):924-935, September 2002.

[2] W. Heinzelman, A. Chandrakasan and H. Balakarishnan, "Energy-Efficient Communication Protocols for Wireless Microsensor Networks," Proceedings of the Hawaaian International Conference on Systems Science, January 2000.

[3] N. Meghanathan, "A Comprehensive Review and Performance Analysis of Data Gathering Algorithms for Wireless Sensor Networks," International Journal of Interdisciplinary Telecommunications and Networking (IJITN), vol. 4, no. 2, pp. 1-29, April-June 2012.

[4] T. H. Cormen, C. E. Leiserson, R. L. Rivest and C. Stein, "Introduction to Algorithms," $3^{\text {rd }}$ Edition, MIT Press, July 2009.

[5] M. Abolhasan, T. Wysocki and E. Dutkiewicz, "A Review of Routing Protocols for Mobile Ad hoc Networks," Ad hoc Networks, vol. 2, no. 1, pp. 1-22, January 2004.

[6] A. J. Viterbi, "CDMA: Principles of Spread Spectrum Communication," 1 st edition, Prentice Hall, April 1995.

[7] T. S. Rappaport, "Wireless Communications: Principles and Practice," $2^{\text {nd }}$ edition, Prentice Hall, January 2002.

[8] C. Bettstetter, H. Hartenstein and X. Perez-Costa, "Stochastic Properties of the Random-Way Point Mobility Model," Wireless Networks, pp. 555 - 567, vol. 10, no. 5, Sept. 2004. 